\title{
LA GEOGRAFÍA RURAL Y EL ORDENAMIENTO TERRITORIAL
}

\author{
Luis Marino Santana Rodríguez*
}

\section{Reseña del libro "Geografía Rural y de la Agricultura" de Jaime Vásquez Sánchez Ph.D. (Junio de 2000)}

Hoy más que nunca, Colombia debe volver su mirada hacia el campo. En él es notorio la agudización del conflicto armado, la disminución progresiva de la producción agropecuaria como producto de la apertura económica, los impactos negativos sobre los recursos naturales, el aumento de la pobreza y los problemas de desplazamiento forzado con la consecuente tugurización de las ciudades. La geografía como disciplina tiene mucho que aportar en el estudio de la realidad rural, como pre-requisito para entender lo que allí ocurre, así como para proponer medidas de intervención que redunden en el logro de condiciones dignas y de mayor bienestar.

El libro comprende un trabajo de sistematización que recoge e integra, de muy buena manera, los aspectos más sobresalientes de la actividad agropecuaria a nivel mundial, regional, nacional y local; además, plantea una discusión sobre lo que representa el espacio rural, las diferentes prácticas de intervención que soporta, los conflictos sociales que en él ocurren y la necesidad de implementar, en este sector, estrategias de desarrollo que permitan aminorar las desigualdades sociales y las condiciones de pobreza. Todo ello a través de siete capítulos.

En el primer capítulo, ei texto presenta una discusión de conceptos básicos tales como geografía rural, qué se concibe por "lo rural" y por lo agrario; sobre los espacios explotados, natural y habitado; y sobre las características de la agricultura y su papel frente al desarrollo económico en zonas con diferentes características ambientales.

En relación al primer concepto, aun cuando el autor reconoce que la geografía rural es más amplia que la geografía de la agricultura, concluye que la primera "tiene como propósito explicar los principios que rigen las formaciones espaciales de la agricultura, sus variaciones como actividad

\footnotetext{
"Profesor Departamento de Geografía, Unniversidad del Valle.
} 
económica, lo que supone estudiar los factores y fuerzas de la localización y distribución geográfica de los hechos agrícolas y sus conexiones espaciales; en síntesis los modos de organización espacial de la agricultura."(Pag. 20).

Es cierto que la actividad agrícola y pecuaria constituyen las más preponderantes formas de intervención de lo rural, sin embargo no son las únicas. En éste sentido el autor plantea que "el espacio rural es aquel que se caracteriza por ser un lugar dinámico donde las actividades económicas, distribuidas espacialmente, alcanzan escalas productivas inferiores a las de los centros urbanos dado su empleo extensivo del suelo, su dispersa población y menores índices de poblamiento; es el espacio en donde se conjugan elementos culturales y materiales, en el tejido social ..." Más adelante reconoce "el espacio rural, además de ser objeto de las actividades agropecuarias, forestales y mineras, cumple con nuevas funciones relacionadas con servicios, tales como el conservar la naturaleza, ser sumidero de $\mathrm{CO} 2$, reproductor de agua, mantener espacios verdes y los recursos recreativos, paisajísticos, .." (pág. 23).

De lo anterior se reconoce entonces, que en el espacio rural tiene cabida una serie de actividades y problemas complejos que deben ser abordados por diferentes disciplinas; sin embargo, la geografia rural está llamada a responder a la pregunta, (tal como lo plantea el autor), " Cómo y por qué se organizan los espacios rurales de una determinada manera?".

Después de abordar factores de orden natural y social que confluyen en los espacios para conformar territorios con diferentes potencialidades y limitaciones, pasa a desarrollar las posibilidades de contribución de la agricultura al desarrollo económico, mostrando la distribución de la superficie agraria en el mundo.

Aun cuando los conceptos centrales de la temática son abordados y sustentados suficientemente en el capítulo, hubiese sido importante incorporar una discusión sobre otros, que se prestan a diversas interpretaciones; me refiero a la misma connotación de espacio y territorio, además de región, paisaje, unidad de tierra y uso de la tierra ${ }^{1}$.

El capítulo dos está dedicado a clasificar y caracterizar a nivel general los sistemas agrícolas existentes, dentro de un contexto de región, y basado en variables como tenencia de la tierra, tamaño de las explotaciones, volúmenes

\footnotetext{
${ }^{1}$ Para ampliar el nivel de información sobre estos conceptos se recomienda ver a Santos, Milton. 2000. "La Naturaleza del Espacio. Técnica y Tiempo, Razón y Emoción". Ariel Editores, España. Renato Ortiz. 1998. "Espacio y Territorialidades". En Otro Territorio. Tercer Mundo Editores, Santafé de Bogotá. Delgado, Ovidio 2001. "Discursos sobre el Espacio en la Geografía Contemporánea”. En revista Semestre Geográfico. Volumen 1 No. 1. Bogotá - Colombia. Páginas 23 a 37. Méndez Ricardo y Molinero Fernando 2000. "Espacios y Sociedades: Introducción a la Geografia Regional del Mundo". 6ta edición, Ariel - España. Gil, Carlos J. 2001. "La Geografía y el nuevo Siglo: iSin Espacialidades?". En revista Semestre Geográfico. Volumen 1 No. 1 Bogotá - Colombia. Pag. 38 - 45.
} 
de insumos, fuerza de trabajo empleada, destino de la producción, entre otras. Se destacan la agricultura de subsistencia, de mercado y de plantación. Termina con un análisis sobre fundamentos para entender la localización de las actividades rurales, utilizando para ello, un breve recuento de los modelos clásicos de localización agrícola.

La caracterización de los principales cultivos (arroz, trigo, maíz, plátano, café, caña de azúcar y algodón), a nivel mundial, de América Latina, de Colombia y del Valle del Cauca, se presenta en los capítulos tres a seis; se hace énfasis en los aspectos de países productores, cobertura de siembra, superficie de producción, productividad y comercio. Para el caso de América Latina se logra presentar exitosamente, reconociendo las limitaciones de escala, una caracterización de las condiciones ambientales imperantes: relieve, suelos y clima, en yuxtaposición con las formas sociales de organización y los niveles tecnológicos y de manejo. Para el caso de Colombia, además de los cultivos principales, se hace un análisis sobre la evolución y tipos de agricultura: de economía campesina, de colonización, el de latifundio tradicional y el capitalismo agrario, junto con una descripción pormenorizada de la estructura agraria que ha primado en el país en las últimas cuatro décadas, sustentada con estadística histórica sobre la tenencia de la tierra y el tamaño de las fincas.

Del departamento del Valle del Cauca, además de sus condiciones naturales, demográficas e institucionales relacionadas con lo rural - agrícola, se presentan datos que muestran el nivel de contribución de los sectores económicos -primario, secundario y terciario- al desarrollo económico del Departamento. Se llega también a cuestionar el impacto de las actividades industriales y agropecuarias sobre el ambiente y la homogenización del paisaje "valle" con cultivo de caña de azúcar. $\mathrm{Al}$ respecto, el autor señala que estas actividades "son causantes de un gran desequilibrio ecológico en la región, que los índices de contaminación del río Cauca no se detienen, que reciben por falta de un control adecuado mayores cantidades de aguas residuales, vinazas, aguas azucaradas y desechos industriales en general existe al mismo tiempo una gran fragilidad en las cuencas hidrográficas;..."(páginas 220 - 221).

El autor ha empleado una de las fortalezas históricas de la geografía, cual es el manejo de niveles de detalle o escalas. Se pasa de lo macro a lo micro, pasando por lo meso; es decir de lo general -agricultura mundial- a lo local geografia del Valle del Cauca, sin obviar a América Latina, presentando información de acuerdo con ese nivel de simplificación de la realidad. Lo anterior hace que tenga mayor vigencia una discusión a fondo, sobre el sentido de lo regional y lo local. Ahora bien, con el desarrollo de la globalización es posible que se piense que esos bastiones de la geografia -el territorio y la región- se pierdan o debiliten como consecuencia del desarrollo de los medios de comunicación y la informática; de todas maneras, son conceptos que perdurarán por la necesidad que tenemos los seres humanos de sentirnos 
parte de un territorio, de construir lugares con sentido de pertenencia. ${ }^{2}$

En el séptimo y último capítulo, se aborda el tema de ordenación del territorio rural. Se trata de una "empresa" relativamente nueva en el país, que requiere, para emprender tan necesaria tarea, de importantes aportes no solo de la geografía, sino de otras disciplinas.

Para Vásquez la ordenación del territorio, "es el conjunto de acciones de planificación o intervención voluntaria sobre el territorio encaminadas al logro de un sistema espacial organizado y equilibrado, desde un punto de vista ético-espacial específico, donde desaparezcan las grandes desigualdades..." (pág. 225). Reconoce que existe una diversidad de conceptos sobre el particular que merecen ser discutidos y precisados, por lo tanto queda por establecer diferencias sobre, por ejemplo, organización del espacio, organización del territorio, ordenamiento territorial, etc. Esta diversidad de interpretaciones se agrava, en buena parte, ya que alrededor del ordenamiento territorial confluyen muchas disciplinas, cada una de las cuales aborda la temática desde su campo de acción o conocimiento.

Toma partido frente a la problemática actual del ordenamiento territorial. Reconoce que toda esta afluencia hacia el OT se inicia desde el poder central, con unos lineamientos muy generales e incluso abstractos; desconociendo en muchos casos las particularidades de los territorios y regiones, y las necesidades y pretensiones de los pobladores. (Énfasis agregado). Es así como se plantea que se requiere de unas metodologías y técnicas para alcanzar los fines propuestos, al igual que instrumentos, insumos, equipos de trabajo y financiamiento adecuados para implementar las medidas que resulten del proceso de toma de decisiones.

Con mayor detalle, el autor enfatiza sobre dos aspectos: La naturaleza del ordenamiento del medio rural y la realidad social que impera en el área rural.

Con relación al primero, muestra como el medio rural ha estado en desventaja frente a lo urbano; mayormente se planifica e interviene lo rural desde una mirada urbana, para su beneficio exclusivo. Existen estatutos de uso del suelo urbano, más no para lo rural. En un sistema territorial interactúan los subsistemas "medio físico o natural, la población con sus distintas actividades, el poblamiento y la configuración de los asentamientos humanos y el marco legal institucional vigente" (pág. 234). Agregaría que cada población tiene una historia y una cultura, que hacen que las miradas frente al territorio sean variadas y por tanto las decisiones a implementar surgirán a partir de un proceso técnico - científico participativo y concertado.

Instrumentos únicos no tendrían cabida para estudiar distintas realidades

${ }^{2}$ Gil, Carlos J. 2001. La Geografia y el nuevo Siglo: ¿Sin Espacialidades? En revista Semestre Geográfico. Volumen 1 No. 1 . Bogotá - Colombia. Pag. 39. 
territoriales y expectativas de la población. En la página 234, se dice "en un territorio y una sociedad tan fragmentada como la nuestra, no es conveniente hablar de una metodología específica para el desarrollo de los planes de ordenamiento territorial, sino de derroteros o lineamientos generales que se apliquen a lugares específicos de acuerdo con las particularidades e historias de cada uno de ellos." Por lo tanto, hubiese sido importante que se incluyera en el libro un subtema que tratase sobre instrumentos o métodos para abordar el ordenamiento territorial en las zonas rurales, donde por su naturaleza, el proceso debe partir por conocer las potencialidades y limitaciones de las unidades de tierra para usos específicos, los que posteriormente deben ser seleccionados teniendo en cuenta "factores económicos, sociales, culturales, políticos y otros que no sean inherentes a las características biofísicas del sitio". $^{3}$

En Richters ${ }^{4}$, se introduce el concepto manejo del uso de la tierra, el cual, a mi manera de ver, lo podemos considerar como sinónimo al de ordenamiento territorial; este autor concibe el manejo del uso de la tierra como "una actividad que debe ser realizada por el Estado, con la participación y concertación de la comunidad, cuyo fin es la determinación, establecimiento y mantenimiento de una combinación de sistemas de uso, socio-económicamente relevantes, de manera que se garantice el bienestar para la comunidad y la sostenibilidad del recurso tierra. El resultado del manejo debe ser la ordenación del espacio, y, por lo tanto, debe ser un proceso cíclico, en donde exista la posibilidad de hacer todos los ajustes que sean necesarios, tomando como base no solo el ambiente físico, sino también las realidades económica, política, social y cultural".

La perentoriedad que tuvo la Ley 388 de 1997, para la elaboración de planes de ordenamiento territorial municipales, no permitió que el proceso se realizara con las mejores bases filosóficas y/o técnica - científica y de manera concertada. Lo que se vislumbra es que los municipios en su gran mayoría, tuvieron como fin el plan mismo, para cumplir con la Ley, y no la verdadera ordenación del espacio que debe darse en un proceso participativo a largo plazo.

Ahora bien, implementar decisiones sobre "sistemas de usos socioeconómicamente relevantes" $\mathrm{u}$ ordenamiento del territorio rural, pasa por resolver o articular la realidad social del país; es decir el segundo punto del énfasis de este capítulo. El profesor Vásquez muy bien lo señala en la página 240 cuando dice "Mantener nuestra frágil memoria no significa negar una realidad social cada vez más compleja en el área rural, ocasionada por la

${ }^{3}$ Komives, R.; Lucke, O. y Richters, E. 1985. Notas sobre el Uso de la Tierra. Centro Agronómico Tropical para la Investigación y la Enseñanza - CATIE- Turrialba, Costa Rica).

${ }^{4}$ Richters, E. 1995. Metodología para la planificación del Uso de la Tierra en América Tropical. Instituto Interamericano de Cooperación Agrícola -IICA. San José, Costa Rica. 


\section{Entorno Geográfico}

creciente agudización del conflicto social armado..". En la página siguiente plantea "no se puede cristalizar un reordenamiento del territorio en el área rural del país, si no se logra profundizar en el proceso de paz, que garantice condiciones reales para que se den los cambios estructurales que requiere el campo ..." Es claro para cualquier persona que, en las condiciones actuales de confrontación y dominio territorial, la implementación de políticas contenidas en los planes de ordenamiento es un imposible en la mayoría de los municipios del país. Ordenar el territorio rural conlleva tratar de manera integral en el tiempo y/o espacio el conflicto armado, proceso de paz, proceso de planificación del uso de la tierra ${ }^{5}$, descentralización administrativa y recursos.

Termina el autor diciendo que "Tomando todos estos elementos activos y pasivos de la realidad nacional, se puede abrir el camino para sortear de la mejor manera una salida que permita construir un nuevo orden social, económico y político más equilibrado ..." (Pág. 245.)

La etapa en la que se encuentran los planes de ordenamiento territorial municipales -POTs-, amerita que los académicos acompañen el proceso de evaluación de los mismos; que sigan aportando elementos o explicaciones filosóficos y técnico- científico de la realidad nacional, a sabiendas de que los problemas de violencia, conflicto armado y desplazamiento forzado del campo tendrán que irse resolviendo. Lo que plantea el Profesor Vásquez en este capítulo, es un buen insumo para la discusión sobre el ordenamiento territorial que debe imperar en el proceso de toma de decisiones e implementación de las mismas.

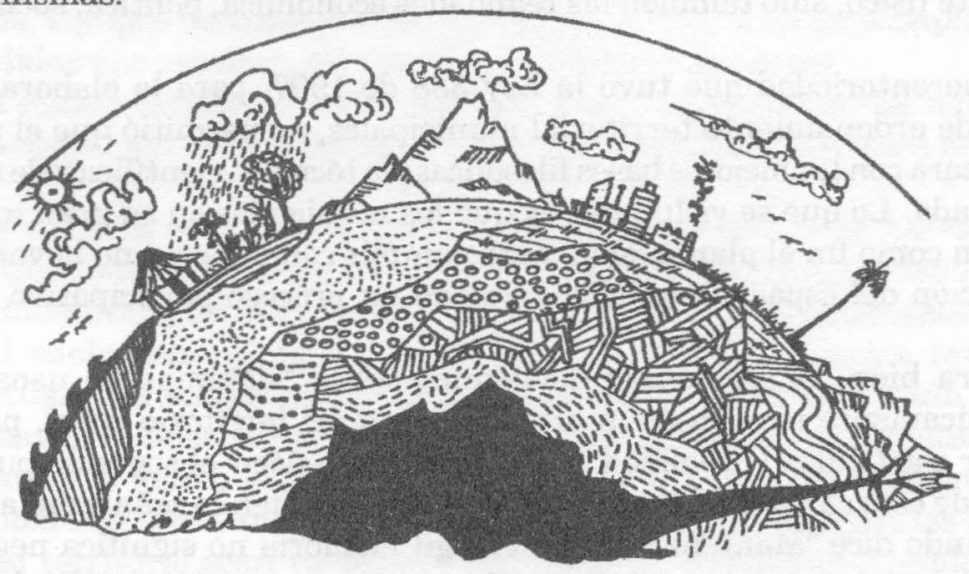

${ }_{5}^{5}$ Tiene que ver con la determinación y en menor medida con el establecimiento de sistemas de uso de tierras potencialmente sostenible. Busca orientar a profesionales de entidades del poder político, sobre las decisiones que se deben tomar en el manejo, a fin de lograr los usos más adecuados del territorio, para que se garantice una buena producción, la conservación de los recursos y una buena calidad del ambiente. 